\title{
Psicología social de hechos, de procesos y de proyectos. Objeto y tiempo
}

\section{Social Psychology of Facts, Processes and Projects. Object and Time}

\section{Resumen}

Una dimensión que debe considerarse en la reflexión que proporcionan las ciencias sociales a la sociedad es la temporalidad. En concreto quiero destacar la idea de temporalidad que está implícita en las formas de conocimiento psicosocial. En este artículo propongo que las orientaciones de la psicología social pueden situarse a lo largo de un eje de temporalidad que distingue entre aquéllas orientaciones que asumen el objeto de conocimiento psicosocial como un hecho (es decir ausencia de temporalidad en el objeto, el cambio es una sucesión de hechos independientes encadenados por relaciones de causalidad) y las que asumen el objeto como proceso (es decir en movimiento, cambio permanente). Finalmente y a raíz de esta propuesta propongo una posible psicología social de proyectos, que comentaré al final, como parte del concepto que manejo de psicología social.

Palabras clave: Temporalidad; Psicología Social
Adriana Gil

Universitat Oberta de Catalunya agilj@uoc.edu
A dimension that must be considered, in the reflection that social sciences offer to society, is temporality. I would like to highlight the implicit idea of temporality within the different forms of psychosocial knowledge. In this article I propose that the different types of social psychology orientations can be situated on an axis of temporality which makes a distinction between those orientations that assume that the object of social knowledge is a fact (that is an object without temporality, being change a mere succession of independent facts), and those orientations that assume that their object is a process (that is an object in movement or perpetual change). Finally, I propose a potential social psychology of projects as an essential part of my own concept of what social psychology is.

Keywords: Temporality, Social Psychology

\section{Entrada}

La intrincada sistematización del sentido común que es la psicología social de corte positivista, aún mayoritaria en nuestros tiempos y en nuestras universidades, nos deja el sabor de que todo ya está de alguna manera dicho; si acaso perfeccionar ciertas técnicas para su mayor eficacia y controlar el efecto de variables adyacentes como el medio, la situación y la cultura, se nos presentan como el quehacer que resta. De esta forma, los "nuevos" fenómenos tratan de explicarse a la luz de los presupuestos teóricos ya existentes en la disciplina y lo más que hacemos es encontrar más variables 
que influyen en la que estamos estudiando, mismas que retomamos en forma de acumulación de conocimientos al respecto de nuestro objeto de estudio.

Esta situación tiene que ver con la forma que consideramos la adecuada para la producción de conocimientos; forma parte de una larga discusión el hecho de que sólo el decidir estudiar algo lleva consigo una serie de parámetros, no sólo del que investiga sino de la forma en que se aprehende la realidad. Los métodos actualmente utilizados en la mayor parte de la psicología social, siendo congruentes con el paradigma positivista de ciencia, abordan los procesos psicosociales de forma atemporal, es decir, como hechos, tratando de describir los mecanismos, las tendencias, las características, las correlaciones o las probabilidades de ocurrencia de los fenómenos, sin dar cuenta de sus procesos de constitución, creación y cambio. Así, los procesos psicosociales, han sido tratados por la psicología social como objetos estables en el tiempo, que determinan las conductas de los sujetos y no se ven como la producción de los propios sujetos.

La preparación de un investigador social consistirá normalmente en aprender a dominar las técnicas del cuestionario; los principios del diseño y el análisis de la encuesta; las complejidades de la verificación, regresión y correlación estadística; análisis factorial y quizás hasta programación de computadoras y técnicas similares. Más aún, todas estas técnicas se aprenden y utilizan como las técnicas de un oficio. Así, un problema surgido en el curso de la investigación, planteará la necesidad de elegir la técnica apropiada para realizar la tarea requerida dentro de los limites establecidos: cuestión de considerar detenidamente la eficacia de determinado instrumento de investigación para que nos dé la información deseada. En suma, se trata de utilizar los métodos de investigación como una tecnología.

Esta forma de proceder en la producción del conocimiento, es lo que provocó, en distintos momentos históricos y desde su aparición en el escenario intelectual, una sensación de fracaso por parte de algunos psicólogos sociales ante su incapacidad de ofrecer análisis de la vida social tan convincentes como los ofrecidos por las ciencias naturales respecto del mundo material. Los fenómenos sociales tienen el "defecto" de estar en constante movimiento, de ser procesos relacionados entre sí, por lo que no se agotan en el conocimiento de causas y efectos. Los procesos psicosociales no esperan "razonablemente" a que el desarrollo teórico-académico continúe su rumbo con su respectiva metodología precisa y concreta.

La búsqueda de cientificidad que tan afanosamente se ha perseguido nos ha llevado al punto de estar no digamos ya desvinculados sino en dirección opuesta de la realidad social cotidiana. EI conocimiento, temporalmente hablando y para el positivista, se ha dividido en dos momentos -el presente en el que se encuentra el que investiga y el pasado en el que el fenómeno o el hecho sucedió-. De esta forma, el científico social se encuentra ante hechos consumados o en vías de serlo en términos de su relevancia social y su actualidad; cuando él puede acceder a ellos, lo que puede conocer está fuera de las múltiples articulaciones del contexto social en el que se inscribe.

Pero, ni podemos estudiar la vida social como si las ciencias naturales no existieran, ni tampoco podemos conservar este rasgo inevitable de nuestros antecedentes intelectuales. Por ello, se presenta como necesidad la recuperación de la psicología social como disciplina de la cultura, de la vida cotidiana, depositaria ella, de las necesidades genuinas de la sociedad civil, generadora y constructora de los procesos que estudiamos sin su cualidad, de manera artificial y aislada. La realidad psicosocial no se agota en los contenidos, es necesario elucidar los procesos de 
construcción de realidades que contemplan la afectividad de la sociedad en términos de sus significados.

El dar cuenta de los procesos resulta imposible desde esquemas interpretativos tradicionales y de metodologías que se ubican, temporalmente hablando, en una eternización presente de la explicación que dan de lo social: lo que ahora observamos, es efecto de sus causas pasadas y tenderá a ser igual en lo futuro. Es decir, la realidad es una, y podríamos acceder a ella de forma precisa y controlada como un continuo acumulativo. Lo que por una parte, equivaldría a considerar que los hechos sociales existen con independencia de las prácticas cotidianas que los constituyen y por otra parte (y como consecuencia), también dejaría de lado el papel activo que desempeñan los sujetos para definir los significados de las situaciones sociales en las que están implicados. En éste sentido no partiremos del supuesto de que el trabajo a realizar es fundamentalmente descriptivo-explicativo, como la psicología social de índole positivista ha venido pretendiendo, sino interpretativocomprensivo. La siguiente cuestión por ende, radica en indagar quien decide la validez de nuestras interpretaciones, y aunque parezca atrevido diría que viene dada por todos, es decir por la sociedad misma, en el momento en que dicha interpretación se comprende, se explica, forma parte de las experiencias y adquiere sentido. Nuestros juicios y justificaciones son válidos en virtud de concepciones sustentadas colectivamente respecto a la realidad y al modo en que nos relacionamos con ella.

Con diferentes énfasis, la necesidad de abordar los fenómenos como procesos, como un problema de temporalidad en psicología social, ha sido manifestada en diversas ocasiones. Kenneth Gergen (1973), expuso la condición de ciencia histórica de la psicología social, pero ya diez años antes Eduard Nicol (1941), consideraba indispensable el estudio de la temporalidad como característica y cualidad de la experiencia de los sujetos. Entre los setenta y los noventa del siglo pasado, muchos marcaron un camino a seguir, por ejemplo Miguel Ángel Aguilar y Óscar Cisneros (1990), señalaron como problema de la psicosociología la distancia entre un evento y su análisis, Hugo Zemelman (1987), desde una reflexión epistemológica, resaltó la necesidad de construcción de una epistemología referida a la realidad histórica social como dándose a un pensamiento abierto a la realidad en constante cambio que concierne a las ciencias sociales en general. Finalmente el construccionismo social de autores como Tomás Ibáñez (2001), se convirtió en el marco epistémico y ontológico para la progresiva asunción en psicología social de diferentes métodos que se pueden emparentar bajo el rubro de análisis del discurso (íñiguez, 2003).

En este trabajo, que sigue el camino marcado por estos autores entre muchos otros, argumento como es que la realidad es una construcción social dinámica, a través de la construcción e intercambio de significados en el proceso de comunicación que se crea y recrea en la sociedad. Pretendo resaltar la importancia de estudiar los fenómenos considerados dentro del campo de la psicología social en su carácter dinámico, de estar en constante cambio y construcción por parte de los actores sociales. Explico como éste movimiento presente en la realidad no puede ser abordado por medio de métodos que derivan de orientaciones teóricas que suponen una realidad estática, lista para ser aprehendida por el investigador. Para ello, propongo que las orientaciones de la psicología social pueden situarse a lo largo de un eje de temporalidad que distingue entre aquéllas orientaciones que asumen el objeto de conocimiento psicosocial como un hecho y las que asumen el objeto como proceso. 
Finalmente y a raíz de esta propuesta considero una posible psicología social de proyectos ${ }^{1}$. En el minucioso estudio positivista y neo-positivista de las conductas, no aparecía ni por casualidad lo que ya el traductor al español de George Herbert Mead denominaba el espíritu, ni la afectividad. Los símbolos con sus significados. Aquello que no nos convierte en ingenieros del comportamiento. Los elementos que constituyen la realidad simbólica, que es a la que podemos acceder, esta comunicación que expresa, intercambia e interpreta símbolos y significados, contiene y da forma a la posibilidad misma de que la utopía se convierta en realidad social. A que en el presente, se reconozca la capacidad de actuación de los sujetos en la construcción de sus proyectos. El conocimiento mismo no debe plantearse ya solo como reconstrucción de lo pasado, sino como apropiación del futuro como lo que en el presente se nos muestra como posibilidad: la construcción de un proyecto cuyos contenidos sean significativos y propios de la vida afectiva de la sociedad. Poder recuperar las necesidades, las propuestas, los sentires que la sociedad misma tiene para sí.

Hago referencia a la necesidad de que las formas de pensamiento y razonamiento de la psicología social no confundan el delimitar un objeto con el hacerle perder complejidad. Porque quizá como en el viaje a Itaca lo significativo de la aventura no es tanto el término del viaje como el viaje mismo. El objetivo se cumple por la experiencia de la búsqueda en sí. El sentido se construye en el momento de hacerlo, de vivirlo. La psicología social debe participar explícitamente de esta aventura, no puede fingir ser solamente observadora.

\section{Psicología social de hechos}

Bajo este rubro, ubico la psicología social mainstream, la que empieza en los experimentos decimonónicos, se consolida con el conductismo y se mantiene viva en el cognitivismo. Se caracteriza por conceptualizar la realidad como un acontecimiento independiente de las creencias de las personas que necesita de un método apropiado de comprobación y control. Lo que de forma esquemática constituye la producción de conocimiento científico: a) los "hechos" son "cosas" o "fenómenos" que existen en el mundo, es decir, están ahí, dados, listos para conocerlos y aprehenderlos mediante su observación; b) es necesario atravesar las apariencias, es decir, "registrar" de forma fidedigna lo que se nos presenta en la realidad, a través de un método que nos permita distinguir un "hecho" del que no lo es, porque no cuenta con un método para corroborarlo; c) a medida que el número de hechos conocidos en diferentes situaciones aumenta, podrán hacerse generalizaciones al respecto de las relaciones que los diferentes hechos guardan entre sí y de esta forma podrán inferirse a manera de explicaciones y predicciones el comportamiento de tales hechos en el futuro.

Para esta psicología social, lo que en su día fueron temas posibles de la psicología social como el aprendizaje o socialización, las opiniones, las actitudes, la atracción interpersonal, la influencia, el liderazgo, etc., pasan a ser "hechos", partes de la realidad que esperaron a la psicología social para ser "descubiertas". El caso es que la relación entre hechos que se establece, es una relación causal, o una correlación que aspira en futuras investigaciones a encontrar la causa, sobre la que el investigador analiza lo social como una continuidad que se originó en el pasado y que no tiene posibilidad de transformarse por otras causas o por la acción de los sujetos. Esta visión causal y por lo tanto estática, de la realidad social es lo que denominamos psicología social de los hechos.

\footnotetext{
${ }^{1}$ Esta propuesta se basa a su vez en la propuesta desarrollada en Adriana Gil (1992).
} 
El tiempo es lineal, es un presente eterno. El hecho de que se considere la existencia de un transcurso del tiempo en el que se acumularán más evidencias para la verificación de los hechos que doy por sentados, en nada modifica mi visión de esos hechos, siguen siendo los mismos fenómenos de los cuales quiero poseer cada vez más información, el que tenga que pasar cierto tiempo para conocerlos mejor, para que maduren, también es un hecho.

Para la psicología social de los hechos estos son infinitamente reducibles a pequeñas partes, cada una aportando su pequeño grano de información. Se pueden fotografiar las distintas partes y juntando las fotos se tiene una visión del conjunto, pero, eso sí, siempre estática. Esta forma de estudiar el presente presupone que el pasado también fue igual y que el futuro es predecible porque los hechos seguirán siendo iguales. La noción básica para esta psicología es la de control, se controla el pasado (se describe lo que pasó), se controla el presente (se describe lo que pasa), se controla el futuro (se describe lo que ocurrirá). Esto forma la base de una psicología social ciertamente optimista. Es la psicología social que creía que con su ayuda, se podría diseñar una sociedad mejor, la que creía que sus intervenciones servían inevitablemente para mejorar el estado de las cosas. El tiempo en estas orientaciones es una serie de puntos en fila que constituyen una línea que va del pasado al futuro, sin solución de continuidad, sin sobresaltos, sin desviaciones, pero sobre todo sin significado.

\section{Psicología social de procesos}

Desde esta perspectiva, que hunde sus raíces en la contra-tradición que partió de la decimonónica psicología de los pueblos, pasó por Gabriel Tarde, por Mead y Lev Semenovich Vigotsky, se desarrolló en el interaccionismo simbólico y explosionó en la llamada crisis de la psicología social de los años 70, la realidad social no tiene una dirección predeterminada sino que es construida por los actores sociales a medida que se desarrolla. Ante un proceso se hace difícil hablar de determinaciones, es más fácil hablar de posibilidades (históricas, societales, simbólicas). El proceso no tiene una dirección preestablecida de manera que en cualquier momento se puede transformar su dirección e incluso puede convertirse en otro proceso. El paradigma del proceso es la comunicación, de por sí dinámica e impredecible. Los procesos sociales no tienen una dirección predecible, en términos de que van a dar por consecuencia un resultado o un producto determinado. Las múltiples direcciones que puede tomar un proceso vienen de que un sujeto social es un sujeto en devenir, no es una 'cosa" ya hecha que solamente recibe impactos del medio exterior, está dotado de subjetividad, interpreta los símbolos compartidos con otros sujetos intersubjetivamente, les confiere significados, les da sentido y puede optar por transformarlos.

La comunicación tiene lugar a través de los símbolos, no únicamente los del lenguaje verbal, sino también de las imágenes, los afectos, la música o los movimientos corporales. Pero todos estos procesos son simbólicos: la interacción, propuesta por la psicología social de los hechos como el efecto de otros en la conducta de un individuo, es sólo una pequeña parte de la comunicación y vista desde su proceso es algo diferente. La comunicación es un universo de símbolos y significados del que disponen los sujetos para definir una realidad común, no hay un efecto de los otros en uno, sino un intercambio de símbolos, cuyo significado puede cambiar según el sentido y de acuerdo al sentido pueden crearse nuevos significados para un mismo símbolo e incluso nuevos símbolos para los significados que aún no tienen nombre. De la primera forma, los otros son la causa del efecto (la conducta), que la interacción con ellos provoca en un individuo, donde lo que no se sabe es que ocurre en la interacción. De la segunda forma, la interacción no es la línea que une las causas y los efectos o unos sujetos con otros, sino la interacción de los símbolos, significados y sentidos. Cuando 
los individuos o los grupos interactúan, intercambian símbolos y les confieren un significado, pero el sentido es el tercer elemento que no está con templado en la psicología social de los hechos, que es lo que permite que la interacción se refiera a una realidad y no otra y provoque determinada "conducta" en los sujetos. La realidad no es un mundo de cosas, de hechos y de sujetos, la realidad que podemos conocer es una realidad significativa, simbólica, no existe una correspondencia de los símbolos con la realidad, existen múltiples significados para los símbolos y sus sentidos correspondientes.

Respecto a la temporalidad, para la psicología social de procesos el tiempo no es lineal, aunque efectivamente se sigue moviendo en un único tiempo: el presente. Pero el presente es un tiempo de construcción compartida, de reconstrucción y reinvención del pasado y de proyectar el futuro. El tiempo no es lineal porque el presente no es el punto de una línea desplegada de tiempo, que tiene un hacia atrás y un hacia adelante, donde podemos ubicar cuantitativamente los puntos más pasados o los menos futuros. Ciertamente existen experiencias pasadas que son más lejanas que otras, pero no quiere decir que las más cercanas sean temporalmente recientes, porque esto implica seguir considerando una noción de temporalidad lineal, uniforme, neutra y cuantitativa. La proximidad o lejanía, es decir lo que consideramos pasado o futuro está en función de lo que signifiquen éstos en nuestro momento actual. Lo próximo a nuestro presente puede ser aquello que cronológicamente ubicamos muy atrás o que por estar justamente ubicado en tiempo muy lejano ni siquiera recordemos su ubicación cronológica, y también es posible que lo que consideramos pasado o lejano, sea muy reciente cuantitativamente hablando. El hecho que la temporalidad pase a ser significativa en lugar de acumulativa tiene consecuencias muy importantes para la psicología que se puede hacer. Porque obliga a reconsiderar las nociones de causa y efecto y situarlas en el ámbito de la comunicación. De hecho el mismo objeto de la psicología social cambia porque la comunicación simbólica, el lenguaje, la construcción de pasados, presentes y futuros, pasan a ser la prioridad del análisis, y no para su control y predicción, sino solamente para su comprensión. Una comprensión que debe entenderse como un paso en la dirección de una psicología social de proyectos.

\section{La Temporalidad de los Hechos y la Temporalidad de los Procesos}

Hasta aquí, es posible reconocer que tanto la Psicología Social de los Hechos, como la Psicología social de los Procesos, parten del presente para explicar o interpretar los fenómenos sociales, puesto que el presente es el momento en el que se encuentra el investigador y en el que pretende entender algo acerca de la realidad social; sin embargo, no parten del mismo presente, o mejor dicho, no comparten la misma noción de presente. Para la primera psicología social, el presente se concreta en una repetición de hechos pasados que lo determinan, que lo causan, la realidad social está compuesta de hechos relacionados entre sí de manera causal y la forma adecuada de conocerlos es describiendo objetivamente el funcionamiento, las relaciones y los componentes de una realidad acabada. Para la psicología social de procesos, el presente es un momento de construcción de la realidad mediante el proceso de comunicación, mismo que genera sentidos que mediante su interpretación, esta psicología puede hacer inteligible que el presente es una realidad en movimiento. 


\section{La noción de presente en la Psicología Social de los Hechos}

La psicología social de los hechos conviene en estudiar el presente donde los fenómenos sociales tienen lugar, pero que al hacerlo, considera las explicaciones que obtiene, válidas y únicas para toda circunstancia, en calidad de leyes universales y condiciones generales, aplicables a cualquier momento, en las que conocemos cada vez más acerca de algo que siempre ha estado allí, ha sido de la misma forma y funcionará igualmente en lo sucesivo. Esta psicología social, al considerar que la realidad es un conglomerado de hechos, cuyas relaciones son causales, hace de los hechos objetos "fijables en el tiempo", cuya separación y especialización de los elementos que los componen es entendida como una exactitud creciente en el conocimiento. Si nada va a cambiar esencialmente, se pueden estudiar a las partes más pequeñas, con la finalidad de tener un mayor control sobre las verdaderas causas. Decíamos que esto da por resultado fotografías de los hechos sociales; se obtienen piezas de la realidad social que se analizan por separado, como si cada una tuviera un quantum o un byte de sentido. Cada investigación en psicología social, se vuelve un estudio fotográfico de algún fenómeno, sacando distintas tomas, en distintos ángulos, mostrando cualquier cantidad de aspectos, y como si al juntar todas las fotografías hiciéramos un gran fotomural, teniendo a la disposición del público todo lo que quiera saber acerca de lo social. Se puede retomar cualquier punto en la línea de los hechos y estudiarlo, en cada cual están sus causas y sus efectos y a lo que se aspira, es a mejorar los métodos, que cada vez más finos, nos permiten describir con mayor fidelidad el presente de la realidad social, que en general es la misma siempre, los detalles no cuentan, solo "aparentan".

El considerar los hechos, la experiencia y la vida social desde esta noción de presente, implica hacerlos un objeto, que es exactamente igual en el pasado, el futuro y el presente, retomando a éste último como constancia de su estructura, comportamiento e inmutabilidad, implica también que en este hoy, no cabe nada, es sólo una línea divisoria entre el antes y el después sin contenido. El presente donde encontramos ciertas causas, ya es pasado, dará lugar a los efectos que se concreten en el futuro y a su vez este presente estuvo determinado por las causas que encontramos en el pasado, el futuro se considera como un presente no muy lejano y no desconocido, en virtud de que los hechos se repetirán nuevamente y el pasado se considera como el presente que fue y los hechos de los que pudo dar cuenta, donde conocíamos un poco menos de lo que nuestro presente nos permite ver; una vez que se encuentran las causas y los efectos generales, los hechos más importantes, no hay temporalidad que valga, podemos predecir, controlar, manipular, y los hechos aparecerán en cualquier momento, independientes de toda contingencia.

Todo lo que estudia la psicología social de los hechos, lo hace en un presente eterno, aunque se trate de un fenómeno que ya pasó, lo ve y lo estudia desde su punto de vista actual, desde "el único punto de vista científico". Si va hacia el pasado de ese fenómeno, sólo ve lo que desde el presente puede ver, considerando la "historia" de ese objeto como una madeja de hilo que va desenredándose linealmente, e inevitablemente comienza en una punta y termina en otra, su futuro; el punto de vista científico siempre parte de una realidad acabada.

Cuando se piensa en el tiempo en este sentido, pensamos en un continuo homogéneo en el que los hechos, los pensamientos, lo cotidiano, se alinean y yuxtaponen, sin otro criterio de organización que el de un número de calendario: el 6 de enero, el 5 y el 14 de febrero, el 30 de abril, el 10 de mayo, los días de quincena, el 12 de octubre, el 2 de noviembre, el 24 y el 31 de diciembre, un año, y así sucesivamente; la temporalidad para la psicología social de los hechos es un hecho más, algo que va a suceder siempre y que mantendrá en continua evolución los elementos que también siempre, 
conformarán la realidad social (por ejemplo que los individuos siempre interactúan y se influyen unos a otros mediante esta interacción). Sin embargo, esta multiplicidad de sucesos en el tiempo no puede tomar el aspecto de un número o de un período sin el intermediario de alguna representación simbólica. El 6 de enero o el 10 de mayo no significan una sola cosa como supondría la psicología social de los hechos, hay grupos para los que ni siquiera significan nada, o hay períodos en los que no significan nada y períodos donde son muy importantes las fechas; tienen más de una interpretación, de acuerdo con el momento, los sujetos y la intención con que se formule ésta.

En pocas palabras, para la psicología social de los hechos, el tiempo como cualidad de un proceso, la temporalidad que es constitutiva de él, no existe, desde el momento en que su propio quehacer no lo considera un proceso; la temporalidad es algo dado por sentado, es un hecho más, que siempre está presente y que no puede cambiar los fenómenos estudiados, es una duración en la que tendrán lugar los sucesos que ya sabemos que tendrán lugar. La presencia de un hecho después de otro, permite que se atribuya el segundo al primero y que se encuentre una relación causal entre los dos. Sólo así se entiende que la ciencia que le da origen, la psicología, tenga una disciplina como la psicología del desarrollo, que estudia cada una de las fases por las que necesaria mente tiene que transitar un ser humano, niñez, pubertad, adolescencia, adultez, vejez, como si los procesos psicológicos fueran inherentes a la cronología del sujeto o como si no pudiera ningún individuo salirse de este continuo de evolución, tal vez al decidir entrar a la guerrilla y no pasar por los períodos de adultez y de vejez. La psicología social de los hechos hace lo mismo, pero en su carácter de "social", habla del nacimiento, crecimiento y muerte de un grupo por ejemplo, o divide "aleatoriamente" grupos de hombres y mujeres o grupos de "clases sociales", como si estas categorías se constituyeran en un grupo por sí mismo, sin pensar en los sentidos que comparten o que no comparten. Sin embargo, aunque el presente sea el momento en el que podemos interpretar lo social, la noción del mismo y por lo tanto la de temporalidad se requieren más allá de la relación causal. Al concebir la realidad social como un cúmulo de hechos, la psicología social que se ocupa de éstos, finiquita los procesos, los hechos que estudia ya no pueden continuar porque ya están terminados; justo en este momento es donde la psicología social de los hechos, pierde una riqueza enorme de los fenómenos que estudia: sus procesos (p.e. la hora del "chisme" donde las amas de casa hablan de sus preferencias hacia un partido y del porqué de estas preferencias).

\section{La noción de presente en la Psicología Social de Procesos}

La noción de presente en la psicología social de procesos, es la de un presente como momento de construcción, ahí el pasado no es asunto muerto, se reconstruye y se resignifica o se rescatan aquéllos elementos que no se habían considerado, se vuelve a construir; el futuro se está construyendo con los elementos que ahora consideramos significativos, con las posibilidades que surgen de una nueva interpretación del pasado y del presente, con los distintos proyectos que hagan sentido en el proceso de comunicación. No es una mera repetición de hechos, el presente es justo donde podemos encontrarle una nueva dirección a los que parecían hechos, donde podemos elaborar el proyecto de un futuro diferente, o mejor dicho, donde caben las posibilidades de múltiples futuros, porque no estamos ante hechos consumados sino ante procesos de los que no podemos señalar un final determinado, sólo posibles desenlaces, eso sí unos más posibles que otros, pero solamente posibles, en la medida que lo distintivo de los procesos es su carácter de construidos.

El presente tiene un carácter dinámico. En efecto, el presente lo es porque, lo que llamamos presente es lo que vivimos de un modo actual, no importa si lo que vivimos de forma compartida, pertenece a 
lo que llamamos recuerdos o proyectos, es decir, si pertenecen al "pasado" o al "futuro". Podemos recordar el aroma de un perfume y aunque sea pasado, en ese momento nos provocará una serie de sensaciones como si fuese la primera vez, y cuando escuchamos una canción muy vieja que habíamos aprendido, podemos recordarla casi sin dificultad aunque no la tengamos "presente", ni sepamos cuando, cómo y dónde la escuchamos inicialmente. En ese momento se actualiza. Así también, cuando se dice que en la juventud hay que ver por el futuro o que tal empresa o persona tienen futuro, es porque en nuestro momento presente actuamos, pensamos, construimos, esperamos ese futuro.

El presente no es el punto de una línea desplegada de tiempo, que tiene un hacia atrás y un hacia adelante, donde podemos ubicar cuantitativamente los puntos más pasados o los menos futuros. Ciertamente existen experiencias pasadas que son más lejanas que otras, pero no quiere decir que las más cercanas sean temporalmente recientes, porque esto implica seguir considerando una noción de temporalidad lineal, uniforme, neutra y cuantitativa.

"Todo organismo... se mantiene a sí mismo por medio de relaciones que, extendiéndose tanto hacia adelante como hacia atrás, constituyen una historia del mundo, pero es evidente que ésta surge tan sólo tras la aparición de aquello que proporciona al mundo este valor (el sentido). El pasado consiste en las relaciones de un mundo anterior con un algo emergente, relaciones que consecuentemente han emergido con ese algo." (Mead, 1929: 62; [el paréntesis es mío]).

La proximidad o lejanía, es decir lo que consideramos pasado o futuro está en función de lo que signifiquen éstos en nuestro momento actual. Lo próximo a nuestro presente puede ser aquello que cronológicamente ubicamos muy atrás o que por estar justa mente ubicado en tiempo muy lejano ni siquiera recordemos su ubicación cronológica, y también es posible que lo que consideramos pasado o lejano, sea muy reciente cuantitativamente hablando.

Lo que es cierto es que en términos empíricos distinguimos acontecimientos que son una prolongación hacia atrás de nuestro presente que nos permiten actuar y planear en el presente. Pero que no sabemos distinguir como una consciente referencia al pasado como tal, pasado es ayer, la semana pasada, hace dos horas etc. No podemos fijar un momento preciso en el cual este pasado que forma parte del presente termina, cuando empieza el pasado "pasado" y menos aún cuando es el presente. Justo esta imposibilidad de fijar un límite es lo que consideramos parte del carácter dinámico del presente: el pasado.

Si consideramos que el pasado por sí mismo mantiene en organización cronológica los sucesos, y que olvidamos los que están ubicados más atrás y recordamos mejor los más próximos, (esta noción que permite representar la continuidad de la vida como una línea) entonces para recordar algo de nuestra infancia tendríamos que recorrer cada una de las experiencias sucesivas de aquí hasta el momento aludido. Que esto no es así en la realidad, resulta evidente. Lo que hacemos es un salto de todos los puntos intermedios al pasado que significa algo para nosotros, que hace sentido en nuestra experiencia actual.

"El pasado que construimos desde el punto de vista del nuevo problema de hoy se basa en continuidades que descubrimos en lo que ha surgido y nos sirve hasta que la novedad que surja mañana precise una nueva historia que interprete el nuevo futuro." (Mead, 1929: 60). 
Así tendríamos que dejar de hablar de una temporalidad acumulativa para hablar de la distinción de la temporalidad significativa, es decir, comunicativa, de aquello que necesitamos del pasado para ir reconstruyéndolo en una versión actual, o mejor dicho en los contenidos del pasado que viven en el presente y que por ello se actualizan, cobrando un nuevo sentido, haciendo a un nuevo momento. Lo que determina la proximidad o lejanía de un momento o un tiempo o una experiencia, respecto del presente, es la relación afectiva en términos de imágenes, de emociones, de sentimientos, de sentido y significado que nos producen. Que no es determinable porque no podemos establecer una dirección de ésta relación afectiva, ni su magnitud (más sentido o menos sentido), su dinamismo, su movimiento está en función de nuestros afectos actuales, de su significado presente.

Dicho significado presente, es construido en el proceso de comunicación intersubjetiva:

"la colectividad es su propio símbolo, significado y sentido. Es la colectividad de (hoy) que le interpreta a la colectividad (de mañana) lo que la colectividad (de ayer) dijo, hizo, quiso, perdió, tuvo: el presente que pone en el futuro al pasado; la gente (la sociedad civil) que se dice a sí misma quién es, qué hacer con sus recursos, cuál es su realidad y cómo organizar su coexistencia." (Fernández Christlieb, 1988: 16).

El pasado que en nuestros ejemplos del perfume y la canción parecen aludir a una experiencia individual, íntima, son la actualización de un pasado que también es una construcción social, en primer lugar porque no hablamos de la experiencia de un sujeto aislado, sino de la de alguien que comparte el mundo con otros, en segundo lugar porque es un pasado interpretado comunicativamente y del que resulta un pasado reconstruido, propiedad de todos y de nadie en particular.

"... lo que propone Halbwachs... es que la experiencia actual o pasada no es algo que esté ahí, presto para ser percibido o recordado, sino el producto de una práctica comunicativa de la que surge un mundo objetivo. Todo lo que nos ocurre y todo lo que recordamos lo fijamos y reconstruimos socialmente. La memoria individual no es sino la participación en las múltiples memorias colectivas que, a lo largo de nuestra existencia, nos rodean. A su vez, esas memorias no son sino memorias de memorias relacionadas comunicativamente." (Ramos, 1989: 72).

El pasado entonces, no es uno sino varios, de acuerdo a los diferentes sentidos e interpretaciones que se hayan elaborado, de la misma manera en el presente encontramos más de un sentido, y más de un proyecto. Aún más, un mismo participante de la comunicación, puede tener el pasado de su infancia, de su juventud, del club, asociación o banda a la que perteneció, el presente de su hogar, de su trabajo, de su organización política, etc., y para cada uno de ellos, comparte con otros un proyecto.

"Así supone Halbwachs que los distintos grupos (formales e informales) van generando, a lo largo del tiempo, un pasado significativo, siempre abierto a reelaboraciones atentas a las solicitudes del presente." (Ramos, 1989: 71).

"Si tenemos la sensación de vivir tiempos distintos, con ritmos propios, con diferentes principios de integración de pasado, presente y futuro, con calendarios diferentes, es porque nos integramos en diferentes grupos sociales. Es la interacción práctico comunicativa de cada uno de estos grupos la que define esa temporalidad en la que nos integramos." (Ramos, 1989: 76). 
Resumiendo, no hay presente sin pasado, y no hay experiencia que no sea presente, y si ella no es presente, es recuerdo de una pasada y entonces también ella es presente, pues consiste en el recuerdo actual de la pasada. Y si no es expresión del pasado podrá ser una anticipación del futuro, una proyección del presente hacia un después que no llega todavía, la cual tampoco se da sino en el presente. Lo constante es el presente.

Así como hablamos de un pasado, todos hablamos de un mañana o de un después: un futuro, sin embargo no hablamos de la noción común de que al fin de semana sigue un lunes, o que después del capitalismo sigue el socialismo. No es una consecuencia lógica, a manera de causa-efecto de vivir el presente, sino que dado que éste se constituye por el estar haciendo (aunque sea estar haciendo nada), el futuro se convierte en una posibilidad, en una expectativa sobre la cual forjamos el presente. Una posibilidad que no tiene realidad alguna (contenido presente), sino que es un propósito, un proyecto. Sin esto no habría motivo para ser o para seguir haciendo y ésta posibilidad es creada en el presente de manera indeterminada, incierta, la única certeza es que algo va a pasar, cualquier cosa que sea una posibilidad es futuro.

Por este motivo, en sentido literal el futuro no es nada, es decir, todavía no es. El futuro se presenta como una serie de posibilidades. Para una sociedad éstas se presentan como un horizonte de acción marcado por sus recursos simbólicos, su capacidad para nombrar, representar y dotar de significado a los sucesos cotidianos, donde las acciones individuales y colectivas encuentran su ubicación y son comprensibles (Aguilar y Cisneros, 1990); el futuro se reconoce como construcción social, parte de la experiencia pasada y de la realidad presente que sólo se puede definir en la medida misma del esfuerzo por construirla: su futuro.

"Solo en el futuro podemos reconstruir lo que ha surgido a partir de las continuidades que le descubramos con todo lo que ha ocurrido anteriormente; y conseguimos la base para esa reconstrucción proyectando hacia atrás en la historia las continuidades recién descubiertas." (Mead, 1929: 61).

El presente para la psicología social de procesos, es pues, un momento de posibilidades en potencia; en potencia porque sus símbolos, significados y sentidos, pueden ser reconstruidos o construidos, tanto para la reelaboración de un pasado, como para la construcción de un futuro, es el momento en el que a través del proceso de comunicación se construyen las posibles interpretaciones que la sociedad elabora para sí misma, de acuerdo a lo que le resulte significativo; no es el punto que divide el pasado y el futuro sin contenido, sino el momento cualificado que contiene el sentido de ambos de dos formas. La noción de presente comunicativo de la psicología social de procesos, es posible en primer lugar, porque su consideración de la realidad es la de una realidad en movimiento (el movimiento temporal del proceso comunicativo), susceptible de transformación por los sujetos sociales, transformación que tiene lugar en la interacción de símbolos y significados, más allá de la interacción entre individuos, que es la que considera como real la psicología social de los hechos. De esta forma, la realidad que puede conocer la psicología social de procesos, es una realidad simbólica, realidad de la que puede dar cuenta mediante la interpretación de los sentidos que están en juego en el momento presente en el que el investigador narra los procesos sociales. En segundo lugar, y por lo anterior, el tipo de relaciones que busca la psicología social de procesos en el presente, son las relaciones acordadas en el proceso de comunicación (cfr. Habermas y Apel), a diferencia de las relaciones causales que contiene el presente informativo de la psicología social de los hechos. Múltiples relaciones acordadas que aluden a los diferentes pasados y proyectos que consideran significativos los grupos sociales y de los cuales ésta psicología social como parte de su propio 
proceso comunicativo de conocimiento, participa de su construcción mediante el hacer explícitos los sentidos del proceso social ( $\mathrm{y}$ de su disciplina como parte de él), que están puestos en palabras o en afectos en la vida cotidiana, colaborando así en la construcción y en el reconocimiento de todas las posibilidades que la sociedad tiene para sí.

Así pues, el lenguaje como sistema simbólico de la comunicación, es un depósito de significados que pueden hacerse presentes en cualquier momento y que mediante sus interpretaciones, hechas con lenguaje, reconstruyen un pasado que resulte significativo. Pero el pasado se compone de lo que fue, de lo que pudo ser y no fue y de lo que ahora sabemos que pudo ser y no elegimos, que se articulan en el presente con el sentido que de cada uno de ellos la sociedad se forma y que sabe. Los otros sistemas simbólicos que atienden a las emociones, sentimientos y afectividad de la sociedad, contienen en su comunicación, las posibilidades que no se consideraron para lo que cristalizó como pasado, los proyectos de la gente que aún no están puestos en palabras, y que expresan las potenciales subjetividades sociales que pueden dar una nueva dirección a los procesos, que fluyen en forma de revueltas, manifestaciones, movimientos de masas, punks, etc. Si el sentido del presente se entiende en su esfuerzo por construirlo, se hace necesario explorar sus posibilidades, se hace necesaria una psicología social de proyectos.

Así pues, la forma en que es considerado el tiempo, la temporalidad, por la psicología social de los hechos, es decir, como un hecho más, como mero transcurso que dará por resultado el hecho tal y como se concibe por la disciplina de manera teórica, y verificado de manera empírica; conduce a la obtención de un conocimiento que hará de la sociedad una sociedad predecible y controlable, un hecho. Sin embargo, este argumento no es congruente ni para la misma psicología social de los hechos, a saber, cien o doscientos años de psicología social empiricista, precisa y prometedora, no han hecho, en definitiva, una mejor sociedad, nada permite suponer que sólo les falta tiempo.

Ante la psicología social de verificación de hechos finiquitados (cfr. Gergen, 1973), la psicología social de procesos puede ofrecer una psicología social de construcción de proyectos, esto es, en lugar de investigar las actitudes favorables para votar por los partidos de derecha (siguiendo con nuestro socorrido ejemplo de las amas de casa), podemos echar a andar y/o recuperar los procesos que permitan que gane tal partido, de acuerdo con los sentidos compartidos por los grupos que se conforman alrededor de éstos. Lo anterior no quiere decir que podamos asegurar que tal partido gane, o que los proyectos que podamos hacer inteligibles se cumplan, pero sí que mediante éstos, se es consecuente con el presente comunicativo que la psicología social de procesos concibe y de "paso" se revitaliza la psicología social. La consideración de la temporalidad como categoría de la psicología social de procesos, recupera para la psicología social el carácter histórico de éstos, alude a su construcción y a la posibilidad de optar de los sujetos sociales, implica también para la disciplina, el reto de explicitar sus compromisos, sus propios proyectos de ciencia y de sociedad, implica también el reto de delimitar en qué medida ese monstruo tan temido por la ciencia objetiva, la subjetividad, tendrá lugar en una concepción diferente de ciencia social.

Ahora trataremos de apuntar algunos elementos para lo que puede ser la psicología social de proyectos. 


\section{Psicología social de proyectos}

Gergen (1973), señala que no se puede predecir lo que cambia y que no se puede controlar aquello que tiene la posibilidad de no dejarse controlar. Este ha sido el sentir hasta ahora: los procesos psicosociales no están definidos de una vez y para siempre ni se encuentran aislados, están relacionados entre sí y son susceptibles de cambiar su rumbo toda vez que así lo decidan los sujetos involucrados en dichos procesos. Veíamos que la noción de presente de la psicología social de procesos considera la realidad como una realidad en movimiento, de ahí que aunque también se ubique en el presente, como momento de interpretación de lo social, es un presente que existe como proceso (no acabado, no lineal, con posibilidades de múltiples direcciones, susceptible de transformación), no es un presente hecho fenómeno el cual pueda predecirse y controlarse y del cual sólo se pueda dar cuenta como "historia de los fenómenos sociales". A partir de la noción de presente de la psicología social de procesos, veremos ahora cómo se configura el presente de la psicología social de proyectos y como dicha noción de presente permite ampliar el horizonte del mismo, al terreno de lo posible. Así pues, recapitulemos sobre los elementos que nos permiten plantear una psicología social de proyectos.

\section{La psicología social de procesos como fundamento de la psicología social de proyectos}

La noción de presente de la psicología social de procesos permite considerar la existencia de la psicología social de proyectos. Recordemos que el presente comunicativo en el que la psicología social de procesos interpreta los procesos psicosociales, es un presente donde los símbolos y significados pueden ser reconstruidos o construidos en los posibles sentidos que los diferentes grupos de la sociedad elaboran, a partir de las situaciones concretas de la vida social, de la vida cotidiana; pertenezcan estos al pasado o al futuro. Hasta ahora, la psicología social de procesos consigue hacer inteligible lo social, a través de la interpretación de los sentidos que se encuentran en el presente comunicativo, que es desde donde el investigador narra los procesos sociales y trata de comprenderlos. Pero ya veíamos que dichos sentidos presentes, están hechos de material simbólico que contiene la cualidad temporal propia de la construcción de los sujetos: el lenguaje -del que nos servimos tanto para la reconstrucción e interpretación de un pasado, como para la objetivación y proyección de un futuro, puesto que es un depósito simbólico capaz de actualizarse en cualquier momento-; los afectos y los otros sistemas simbólicos -que contienen las posibilidades que no se constituyeron como pasado y los deseos, las utopías que se conciben como futuro posible-. Así pues, si la realidad simbólica que conoce la psicología social de procesos está en constante devenir, y manifiesta sus sentidos presentes en la medida en que los construye o resignifica, el presente comunicativo se extiende más allá del proceso que podemos ver o sentir, el presente comunicativo es un proceso abierto a la gama de lo posible, de lo que todavía no es y de lo que no ha sido, de lo que podemos inventar, intuir, de los proyectos. Es en este sentido que el presente comunicativo se vuelve aún más fluido, es el espíritu de la comunicación que se torna un terreno fértil para que la psicología social se piense como psicología social de proyectos. Así pues, la psicología social de proyectos no existe, no aún, es un proyecto también. Pero como proyecto, significa que existen elementos presentes y elementos pasados que se actualizan, que se construyen y reconstruyen dentro del ámbito de la comunicación "científica" y que permiten plantearla, y también como proyecto significa una posibilidad, una expectativa sobre la cual forjamos el presente y con la que podemos reconocer nuevos procesos y nuevas direcciones de éstos; dichos elementos los hemos reconocido en este 
trabajo como la psicología social de los hechos, frente a la cual se construye la de proyectos, y como la psicología social de procesos, en la cual encuentra su fundamento.

\section{De la temporalidad y de lo posible}

Decíamos, que la psicología social de los hechos ha llegado a un punto en el que sus explicaciones de lo social, dejan fuera una riqueza vital de experiencias y procesos que permanecen excluidos por su forma de concebir la realidad social. Es decir, como un conglomerado de hechos relacionados entre sí y cuyas relaciones deben explicarse causalmente y en forma de leyes universales. Lo que está dejando fuera la psicología social de los hechos, es la consideración de lo social como realidad simbólica, que no está dada de una vez y para siempre y que como proceso está abierto al horizonte de lo posible. La aprehensión de la realidad social como proceso, es posible gracias a la consideración de la temporalidad, del movimiento, de la historicidad, como cualidad propia de lo social. Sobre estos dos ejes, el de la temporalidad, y el de lo posible como manifestación de la primera, es que reconocemos la apertura de la psicología social de procesos hacia una psicología social de proyectos, que le permite a esta última descomponer lo "dado por hecho" y apuntar a un enriquecimiento de lo simbólico, ya que en efecto, la psicología social de los hechos no sólo no ha hecho una mejor sociedad con su quehacer científico, sino que se ha ganado un lugar importante en el empobrecimiento simbólico de los recursos que ésta tiene para comprenderse y de su propio ámbito como disciplina. Veamos ahora los "dados por hecho" de la psicología social de los hechos que ya hemos señalado, pero esta vez, a manera de elementos en los cuales la psicología social de proyectos, puede reconocer las oportunidades de ampliar lo que hasta ahora es posible de "observar" como realidad social.

Decíamos que el considerar los fenómenos sociales como hechos, cuyos elementos son perfectamente reconocibles e identificables en cualquier momento, ya como causas, ya como efectos o ya como factores "adyacentes", da por resultado un presente sin contenido, ya que sólo se puede ver en él lo causado por el pasado y lo predecible por el mismo sentido causal, en el futuro; el "dado por hecho" es la suposición de una realidad acabada, en la cual sólo evolucionan los hechos conocidos, realidad que pude manipularse, para reducir el tiempo de evolución de los hechos y para lograr su "aparición" en cualquier momento. Desde este punto de vista, la realidad social tiene una sola interpretación que no es más que "comprobar lo que los hechos demuestran" y tanto ustedes como yo no tenemos más que "aceptar los hechos tal como son", si alguna duda cabe solamente "mírelo usted mismo" y si aún así se puede dudar "a los hechos me remito". Lo que en otras palabras significa que la realidad social es algo "suprahumano" -no resulta de la actividad humana, no es contingente, ni subjetiva, ni variable-, o algo "supracomunicativo" según la psicología social de procesos diría. Pero también según ésta, las frases que citamos arriba, son acuerdos a los que ha llegado la psicología social de los hechos respecto de la realidad social (mejor conocidos como su retórica, Billig, 1987, 1991), en el proceso comunicativo y que llevan implícitos sus decisiones, lo que la coloca como una construcción social más. Ahora, según la psicología social de proyectos, esta interpretación de la realidad es sólo una interpretación y no "la realidad", aún más, es sólo una interpretación posible, ya que los hechos, en tanto que procesos en constante devenir, significan diferentes cosas en contextos distintos donde adquieren su especificidad y donde ya no puede hablarse de un mismo hecho: "alta autoestima" en otro contexto puede significar "egoísmo" o "conformidad" puede ser desde otra interpretación "conducta solidaria" (ver Gergen, 1973). Así pues, considerar la realidad social como hechos relacionados causalmente y vistos desde un sólo ángulo, desde una teoría psicosocial por ejemplo, empobrece las posibilidades de un proceso ya que las 
reduce a una sola. Lo que la psicología social de proyectos haría, sería partir de la noción de realidad en movimiento, de la temporalidad de los procesos y de su carácter de construidos socialmente, para dilucidar los significados posibles de los procesos que interpreta de acuerdo a un sentido específico y los posibles significados que el mismo proceso adquiriría en otros contextos, bajo otros sentidos, con lo cual gana posibilidades tanto su conocimiento de la realidad social, como los propios procesos, ya que las diferentes interpretaciones, los diferentes sentidos que la psicología social de proyectos pueda hacer inteligibles, forman parte del proceso de comunicación de la sociedad y de los recursos de ésta para comprenderse y para elegir una dirección posible de acuerdo a sus proyectos.

"La manera inicial de pensar las relaciones entre diferentes procesos, es confrontar su posibilidad... Esta idea intenta romper con la modalidad de relaciones entre procesos, según ha sido fijada por las diferentes teorías, dado que privilegian una determinada forma de relación sobre otras que pueden adoptar esos mismos procesos en contextos distintos." (Zemelman, 1987: 18 y 19).

Entender que un proceso puede significar varias cosas, incluso cosas que no hemos "visto" como parte de dicho proceso, tiene que ver con el tipo de relaciones entre procesos que podemos imaginar. La psicología social de los hechos, en otro "dado por hecho", entiende a la realidad social, como un conglomerado de hechos, parte de los cuales considera como causas, que en su evolución por el "tiempo" se van relacionando de manera "causal" con otros hechos considerados como efectos, los que a su vez, pasado un "tiempo", serán considerados causas que producirán otros efectos y así sucesivamente. Lo único que hay que hacer es esperar que el tiempo haga evolucionar los hechos naturalmente, para poder "apreciar" las relaciones que los hechos aislados guardan entre sí. Pero esta noción de relaciones entre hechos, no deja de considerar que cada hecho es una cosa aparte, y que no va a cambiar por ser ya la causa, o ya el efecto de otro hecho; lo único que cambia en un hecho, es la ubicación como causa o como efecto, a partir de lo que pretende explicar la psicología social de los hechos, sobre determinado aspecto de la realidad social. Así cuando su interés es explicar otro aspecto, lo que en un momento dado ha considerado causa, en este "nuevo" aspecto se convierte en un efecto, sin que ello interfiera en su calidad de hecho: el liderazgo, es el liderazgo aquí y en China, sólo que aquí es efecto de la personalidad autoritaria de un individuo y en China es la causa de un movimiento social, pero el hecho es el mismo. Lo que la psicología social de procesos diría al respecto, es que los procesos no son "cosas" o "hechos" reconocibles y diferenciados de manera empírica en la realidad en cuanto "objetos" de estudio, sino que son todos los sistemas simbólicos en movimiento, en construcción comunicativa, o en otras palabras, un proceso es todos los procesos relacionados entre sí, otra forma de ver un proceso, de suyo sería una fragmentación de éste. Por lo que la psicología social de proyectos, diría que aquí y en China, en tanto que especificaciones de un proceso, no pasa lo mismo. Lo anterior porque las relaciones entre procesos no son relaciones causales desarrolladas en el "tiempo", son relaciones acordadas en el proceso de comunicación, susceptibles de transformarse por su cualidad "temporal" y que de acuerdo con su sentido presente, adquieren un significado particular, pero no en forma de diferentes hechos, sino como especificaciones del proceso íntegro de la comunicación; así un significado particular, puede cambiar bajo otro sentido, puesto que las relaciones que especifican un proceso no están determinadas de una vez y para siempre:

"...los procesos distinguibles en la realidad no se desvinculan unos de otros, sino en el marco de relaciones necesarias que deben reconstruirse." (Zemelman, 1987: 26). 
"La lógica que debe guiar al establecimiento de las relaciones posibles, no es sin embargo, unívoca... un fenómeno concreto, por ejemplo, la productividad, requiere ser analizado desde diversos ángulos de enfoque... puesto que, por formar parte de una realidad compleja e integrada, el fenómeno sintetiza, de una manera particular, las diferentes dimensiones de la realidad cultural, política, psicosocial." (Zemelman, 1987: 19)

El considerar la realidad social como relaciones causales entre hechos aislados, reduce doblemente las posibilidades de los procesos, primero porque considera un proceso como hecho, con lo cual ya se reducen sus posibilidades, y porque sólo contempla una forma posible de relación entre hechos, la causal, con lo que también se niegan otras relaciones posibles que especifican al proceso en significados diversos. Pero aún tenemos una reducción más de las posibilidades de los procesos, en este sentido, vamos a señalar otro "dado por hecho" de la psicología social de los hechos, que consiste en considerar a éstos como inmutables. Si un hecho "está ahí" en la realidad, independientemente de si lo conocemos o no, quiere decir que ese hecho existe independientemente de nuestras voluntades, deseos, decisiones, opiniones, utopías y preferencias de cualquier tipo; es algo "dado", de ahí la noción de que el psicólogo social ante un hecho, sólo tiene que mostralo tal cual es, mediante los procedimientos adecuados. Recordemos que un hecho sólo evoluciona, no cambia radicalmente, es decir, no se convierte en otro hecho, el que evolucione sólo quiere decir que se vuelve cada vez más "sí mismo". Por eso, si es un hecho que existe el liderazgo, debe poder verse aquí y en China, aunque sea en su primera etapa de personalidad autoritaria, que pasado un tiempo traerá como consecuencia un líder. La psicología social de procesos objetaría esta noción de la realidad social, diciendo que no hay realidad social "afuera" de la comunicación, es decir, independiente de las producciones simbólicas humanas, de los significados que le conferimos intersubjetivamente y que nos permiten comprenderla; porque lo que un hecho significa para nosotros, no parte de una "propiedad" del hecho mismo, sino de la relación que en tanto proceso, tiene con otros procesos y que lo especifican y que podemos interpretar porque compartimos su significado con los demás, significado que acordamos en el proceso de comunicación. Diría pues que la realidad social es una realidad simbólica, y que lo simbólico es tan real como un ladrillo, o como cualquier otra cosa que nos parezca real. Aún más, la psicología social de proyectos diría que la realidad simbólica es tan real que es capaz de transformar la realidad, puesto que si la forma en que la interpretamos cambia, también cambian las cosas que vemos en ella, y tal vez hasta lo que con ellas queremos hacer. Por ejemplo, para la psicología social de los hechos, es un hecho que las mujeres son más fácilmente persuadidas que los hombres, efecto del hecho de que las mujeres son poco audaces, pasivas, sensibles, etc. Pero las mujeres que saben que son blanco fácil de la persuasión, pueden decidir no serlo más y actuar de manera diferente a la de hasta ahora, con lo cual se transformarían las correlaciones en cuanto a la facilidad de persuasión entre hombres y mujeres (Gergen, 1973), provocando una situación diferente a la que se había considerado como un hecho inmutable. De esta forma, la psicología social de proyectos gana posibilidades para los procesos, puesto que no sólo tienen las posibilidades que conocemos hasta ahora, sino las posibilidades de transformar su dirección y convertirse en nuevos procesos, en tanto que, son producidos por el proceso comunicativo, son construidos social mente.

A partir de estos "dados por hecho" que hemos delineado, el presente para la psicología social de proyectos no es un presente sin contenido, es decir, un presente causado por el pasado y predictor por el mismo sentido causal del futuro. Lo posible se vuelve la clave de su presente, en tanto su consideración de una realidad en movimiento, donde los procesos están relacionados entre sí y se 
especifican de manera diversa de acuerdo a diversos sentidos posibles y donde dichos procesos son susceptibles de transformar su dirección o de convertirse en procesos distintos. Esto la coloca en un presente sumamente rico en posibilidades para los procesos que conoce, pero más aún, la coloca ante las posibilidades de que nuevos procesos se construyan, y de "inventarlos" y de enriquecer su comprensión de lo social y de enriquecer los recursos simbólicos de la sociedad para interpretarse y para elegir las direcciones de los procesos que construye.

\section{La noción de presente de la psicología social de proyectos}

Dentro de la noción de presente de la psicología social de los hechos, es posible pensar que el futuro es una realidad previsible y causada por la evolución natural de los hechos, una realidad para la que se acumula conocimiento con el que se pretende tener solución para todas las situaciones futuras y que hará de la sociedad futura, una sociedad precisamente conocida y prácticamente controlable, un hecho. Y siguiendo esta línea, los proyectos se reducen a una planificación del futuro, entendida la planificación como una forma más rápida, más eficaz y menos errónea que la propia evolución de los hechos. Con lo que el presente de la psicología social de los hechos, y su noción de futuro, así como la de proyecto, están reducidas a una sola posibilidad: la de las causas y los efectos y su planificación, o si se entiende mejor, su control. En este sentido, el presente aparece como carente de contenido y sobre todo de posibilidades, de sorpresas, puesto que lo que reconocemos en él está causado por el pasado y el resto es la planificación de un futuro que en cuanto sea presente, no será más que la causa del pasado y la planificación de otro futuro, como vivir el lunes desde el domingo en la tarde. Así pues, sólo tenemos una posible interpretación del pasado, del presente y del futuro, una sola noción del movimiento de la sociedad: un desarrollo hacia adelante, planificado, producto de las causas pasadas y producente de los hechos futuros, en suma una sociedad que cambia para seguir igual, finalmente una realidad acabada.

Nada está más lejos de ser cierto en la noción de presente de la psicología social de proyectos. El presente de ésta podríamos entenderlo como una visión ampliada del presente de la psicología social de procesos; recordemos que éste es entendido como momento de construcción y reconstrucción tanto del pasado como del futuro, en tanto que el presente no es visto como una mera repetición de hechos, ya que la realidad social no tiene una dirección predeterminada por el pasado o por la planificación de un futuro; el presente es justo donde podemos encontrarle una nueva dirección a los que parecían hechos, donde caben las posibilidades de múltiples futuros, en la medida que lo distintivo de los procesos es su carácter de construidos. La psicología social de proyectos a partir de esta noción de presente y de que no comparte los "dados por hecho" de la psicología social de los hechos, encuentra más de una interpretación de la realidad social, o mejor dicho, puede ver varias realidades, porque puede ver varios proyectos; éstos son precisamente la forma por la cual los sujetos se apropian del presente, un proyecto es una nueva interpretación del pasado y del presente, una expectativa que permite verlo diferente, y donde los procesos sociales adquieren sentido y muestran una dirección construida por los sujetos sociales. El futuro en tanto que proyectos, no termina en una planificación, empieza en un presente enriquecido, no con hechos sino con procesos en movimiento, temporalmente construidos. Así pues, lo que está en juego entre una psicología social de los hechos y una psicología social de proyectos no es mera cuestión de nombre, sino el enriquecimiento o el empobrecimiento de la realidad simbólica, de los recursos que la sociedad tiene para comprenderse y para elaborar proyectos y de la propia comprensión de lo social que tiene la psicología social. La clave para que la psicología social de proyectos pueda entender los procesos como temporalmente construidos, con sus múltiples posibilidades, susceptibles de transformarse, la 
encuentra en los proyectos. Toda la vida puede ser vista a la luz de los proyectos, como un constante hacer y deshacerlos: proyectos de trabajo, académicos, de negocios, amorosos, políticos, sociales, económicos, proyectos de vida, aún más, proyectos por inventar, proyectos que encontrarían nuevos símbolos, significados y sentidos a la realidad social, proyectos que enriquecen las posibilidades históricas, culturales, simbólicas de ésta; de ahí que ésta psicología considera al presente como el mejor tiempo: el tiempo de proyectos.

\section{El mejor tiempo: el tiempo de proyectos}

Podríamos preguntarnos qué es lo que distingue a la predicción de la psicología social de los hechos, de un proyecto de la psicología social de proyectos y quizá en este sentido se encuentre la mejor definición de ésta última y de lo que es un proyecto en psicología social. La predicción aunque aparentemente hable de cómo serán las cosas en un futuro, lo hace desde el pasado, es decir, "...la historia de las relaciones pretéritas influye sobre las respuestas actuales... la secuencia precisa de los hechos pasados encierra una clave importante con respecto de la causalidad... un término como 'conformidad' puede referirse a un algo producido o a algo que produce otros resultados [es decir, a una consecuencia de procesos previos o a una causa de efectos ulteriores..." (Hollander, 1967: 34). De esta forma, lo que se predice en el presente para el futuro, está determinado por el pasado de ese fenómeno y por la forma presente en que se le puede observar, sin que se abran posibilidades a dicho fenómeno y sin que se agregue nada al conocimiento presente. Un proyecto, reconstruye un proceso social, considerando lo que no está dado en ese proceso, lo que es posible que contenga éste y que aún no lo consideramos parte de él y en lo que es posible que se con vierta explicitando sus posibles direcciones, un proyecto hace uso de la creatividad, entendida ésta como "la memoria colectiva que cae más adelante" (Fernández Christlieb: comunicación personal) y de esta forma, se enriquece en el presente, el conocimiento de dicho proceso. En este sentido, un proyecto a diferencia de la predicción, no está esperando que lo proyectado se cumpla, que es la finalidad de ésta; un proyecto pretende ampliar la gama de posibilidades de los procesos y explicitar los sentidos a partir de los cuales se construyen, puede incidir en el futuro de la configuración simbólica de la sociedad pero no como planeación del futuro, sino en el enriquecimiento simbólico del presente, es decir, no terminando con los procesos dándolos por hecho, sino colaborando en el proceso comunicativo de la sociedad, en tanto que recupera la capacidad de actuación y transformación de los sujetos sociales.

De esta forma, la labor fundamental de la psicología social de proyectos, es hacer proyectos de los procesos, en lugar de historias de los fenómenos sociales, recuperando así la noción de una realidad en movimiento, construida socialmente. Es decir, reconociendo la cualidad temporal de los procesos y su posibilidad de transformación, ubicándose en el terreno de lo posible para en lugar de verificar un hecho social, inventarlo, para participar en la construcción social de la realidad en lugar de solamente describirla. El mejor tiempo es pues, el tiempo de proyectos, es el momento en el que se está construyendo el presente a través de la apropiación del mismo, en la medida que se elige que dirección pueden seguir los procesos en los que se está involucrado:

"Es gracias a los proyectos que el sujeto establece una relación con la realidad que se apoya en su capacidad de transformar a esa realidad en contenido de una voluntad social, la cual, a su vez, podrá determinar la dirección de los procesos sociales... En este contexto, la apropiación del presente deviene un modo de construir el futuro, y, a la inversa, un proyecto de futuro, protagonizado por un 
sujeto, se transforma en un modo de apropiación del presente." (Zemelman, 1987: 16 y 17$)$.

Si el psicólogo social de proyectos se concibe como un participante más en el proceso comunicativo, en la construcción social de la realidad, entonces debemos entender que los proyectos que elabore no lo convierten en un mesías de la sociedad. Decíamos que la psicología social de proyectos da la visión de varias realidades y de varios proyectos que se configuran a partir de los diferentes sentidos que puedan compartirse en la sociedad, entonces, un proyecto es sólo uno de esos sentidos posibles; si la realidad es entendida como todos los procesos relacionados entre sí y sus especificaciones, un proyecto está inscrito en un sentido particular y en su especificidad, no se trata de que un proyecto sea la versión actual de una visión acabada de la realidad, como la de la psicología social de los hechos, se trata de que un proyecto considere la especificidad de un proceso y su sentido particular, pero a manera de ampliar las potencialidades de dicho proceso y siempre considerando que dicho proyecto es una posibilidad del proceso comunicativo, un proyecto no es la realidad social completa, es sólo una de sus posibilidades:

"El conocimiento del presente no puede organizarse sólo en función de las exigencias de un proyecto en particular, ya que el presente contiene muchas potencialidades que diversos sujetos sociales pueden activar. Un proyecto representa sólo una dirección posible, de manera que antes de elegir un proyecto es necesario reconocer el campo de opciones..." (Zemelman, 1987: 17).

De ahí que como psicólogo social de proyectos, el quehacer de la disciplina sí es un asunto de valores, por una parte, porque uno elige bajo qué sentido compartido elabora un proyecto y qué procesos se quieren potenciar con ello, con lo que se pasa, de ser un observador de lo social, a ser un participante del proceso comunicativo, o si se quiere, a recuperar el papel de ciudadano explícitamente. Por otra parte, porque cualquier proyecto, ya sea que atienda a sentimientos, deseos o utopías que no han sido consideradas como presente, o que pueda ser puesto en lenguaje, o que sea hecho con ambos, es una construcción simbólica, por lo tanto una cuestión de valores.

Decíamos, que en sentido literal el futuro todavía no es, porque se presenta como una serie de posibilidades, es decir como proyectos, decíamos también que todas estas posibilidades son igualmente válidas entre sí pero que existen (valga la redundancia) posibilidades más posibles que otras; ahora bien, qué hace a un proyecto más posible, o por qué se presenta éste y no otro como posibilidad susceptible de hacer sentido en la sociedad en un momento dado, o por qué reconocemos a ciertos proyectos como creíbles y no a toda la gama posible de proyectos. Porque según la psicología social de proyectos, el único límite de éstos es su verosimilitud.

Ahora bien, no debe entenderse verosimilitud como la "verdad" de la psicología social de los hechos, cuando pretende distinguir los "verdaderos hechos" de los que sólo aparentan serlo; la verosimilitud es también una construcción social, una convención, un acuerdo, lo que puede interpretarse socialmente como creíble. No intenta decir lo que ha sucedido, sino lo que podría suceder, esto es, lo posible; es posible que yo quiera demostrar la existencia de los unicornios y su relevancia para la psicología social, pero la existencia de los unicornios y su relevancia, si bien es cierto que es posible, no es necesariamente verosímil, eso depende de si hay sujetos sociales a los que esto les parezca relevante y creíble. Por lo que un proyecto verosímil, es un proyecto creíble en términos de lo que anticipa, de esta forma, la fuerza de un proyecto no radica en la "verdad" sino en la persuasión. Un proyecto "especula" con la posibilidad de un proceso de manera creíble pero interesante, posible, 
pero fuera de lo común, de ahí que pueda ampliar las posibilidades de un proceso. En este sentido lo verosímil tiene que ver con lo mágico, pero no entendido mágico como imposible o como divino, sino mágico como aquello que está fuera del curso ordinario de los hechos (las fantasías, los sueños, los deseos, la imaginación, etc.), es una realidad posible y una posible realidad. Por esta razón, no existe lo verosímil como algo dado, no existe lo verosímil en sí, que pueda ser definido de una vez por todas; en tanto que es una construcción social, transforma también en el proceso comunicativo, su significado, su sentido. Lo verosímil cambia, como cambia la realidad y en tanto que la realidad cambia por la acción de los sujetos sociales, también lo verosímil cambia de acuerdo a lo que pueda ser interpretado como tal. Por ello, la psicología social de proyectos no trata de conocer objetivamente la realidad social y describirla, trata de inventar un proceso, a través de un proyecto que sea verosímil.

\section{Salida: ¿El objeto de la psicología social de proyectos?}

En lugar de ir a buscar el objeto a estudiar en la psicología social a través de las teorías y de lo dado por sentado, debe construirse, buscarse en la realidad simbólica en el terreno de lo posible, en lo que es susceptible de hacer sentido en los sujetos, en lo que pertenece al terreno de los afectos y en lo que concierne al lenguaje capaz de resignificar los símbolos. La psicología social, como psicología social de proyectos debe intuir las relaciones posibles entre los procesos, debe inventar sus "objetos" con el único límite que marca la verosimilitud. El ejemplo que tengo más a la mano es la propia Psicología Social de Proyectos, es un proyecto que hasta donde yo alcanzo a ver, es bastante verosímil y que amplia la gama de posibilidades de comprensión de lo social. Cuando hemos hablado del quehacer de la psicología social como Psicología Social de los hechos, hemos remarcado con este nombre su noción de realidad, de lo social y del conocimiento que produce, pero aunque este nombre nos ha permitido resaltarlo, sólo hemos hecho hasta ahí, psicología social de los hechos, en el sentido de que hemos verificado lo que existe como psicología social, hemos verificado un hecho. En cuanto nos ubicamos en la psicología social de procesos, en su noción de realidad en movimiento y en la posibilidad de transformación de los procesos, hemos podido construir la noción de una psicología social de proyectos, hemos "inventado" una forma de hacer inteligibles las relaciones entre procesos y transformado la visión de los mismos a través de un proyecto, ahora sólo esperamos que éste, resulte verosímil para la psicología social.

Si la psicología social puede reconocer a la sociedad como espíritu comunicativo, como comunicación en devenir, tiene ella misma la opción de construirse como un espíritu también, un espíritu que comprende y enriquece a través de proyectos. Tiene para sí las posibilidades de ser un espíritu crítico.

\section{Referencias}

Aguilar, Miguel Ángel y Cisneros, César. (1990). La continuidad del presente en psicología social y psicología política. Sociológica, 5, 14, 63-76.

Billig, Michael. (1987). Arguing and Thinking. A rhetorical approach to social psychology. Cambridge: Cambridge University Press.

Billig, Michael. (1991). Ideology and Opinions. Studies in Rhetorical Psychology. Londres: Sage. 
Fernández Christlieb, Pablo. (1988). Psicología social, intersubjetividad y psicología colectiva, Mexico DF: Laboratorio de Psicología Social, U.N.A.M.

Gil, Adriana. (1992). Las posibilidades de un espíritu crítico. Tesis de Licenciatura. Facultad de Psicología. Universidad Nacional Autónoma de México.

Gergen, Kenneth (1973). Social Psychology as History. Journal of Personality and Social Psychology , 26, 309-320 (trad. al español en 1998 como Psicología Social como Historia. Revista Anthropos, 177, 39-49.)

Hollander, Edwin P. (1967). Principios y métodos de psicología social. Buenos Aires: Amorrortu, 1982.

Ibáñez, Tomás. (2001). Municiones para disidentes. Realidad-Verdad-Política. Barcelona: Gedisa.

Iñiguez, Lupicinio. (Ed.) (2003). Análisis del discurso. Manual para las ciencias sociales. Barcelona: Editorial UOC.

Mead, George Herbert. (1929). La naturaleza del pasado. Revista de Occidente, 100, 51-62.

Nicol, Eduard (1941). Psicología de las situaciones vitales. México- Buenos Aires: Fondo de Cultura Económica, 1963.

Ramos, Ramón. (1989). Maurice Halbwachs y la memoria colectiva. Revista de Occidente, 100, 6381.

Zemelman, Hugo (1987). Conocimiento y Sujetos Sociales. México: Colegio de México.

\section{Historia editorial}

Recibido: 03/10/2005

Aceptado: 21/04/2006

\section{Formato de citación}

Gil, Adriana. (2006). Psicología social de hechos, de procesos y de proyectos. Objeto y tiempo. Athenea Digital, 9, 78-99. Disponible en http://antalya.uab.es/athenea/num9/gil.pdf.

Adriana Gil Juárez. Licenciada en Psicología por la Universidad Nacional Autónoma de México. Magíster y Doctora en Psicología Social por la Universidad Autónoma de Barcelona. Es Profesora Coordinadora del Área Social y Cultural en los Estudios de Psicología y Ciencias de la Educación de la Universitat Oberta de Catalunya. Dirige el grupo de investigación JovenTIC, que estudia las dinámicas de consumo de las TIC y su relación con la identidad de los adolescentes y el grupo ermeneuTIC: Estudios e Investigación Metodológica de los Nuevos Espacios y Usos de las Tecnologías de la Información y la Comunicación. 


\section{(C)}

Este texto está protegido por una licencia Creative Commons.

Usted es libre de copiar, distribuir y comunicar públicamente la obra bajo las siguientes condiciones:

Reconocimiento: Debe reconocer y citar al autor original.

No comercial. No puede utilizar esta obra para fines comerciales.

Sin obras derivadas. No se puede alterar, transformar, o generar una obra derivada a partir de esta obra.

\section{$\underline{\text { Resumen de licencia }}$}

$\underline{\text { Texto completo de la licencia }}$ 